ISSN 2693-2504

\title{
Robots: Aware Headway and Viable Recovery
}

\author{
Journal of Bioscience \& Biomedical Engineering
}

Case Report

Rinaldo C. Michelini di San Martino

DIMEC - UNIVERSITY OF GENOVA - ITALY

\author{
*Correspondence authors \\ Rinaldo C. Michelini di San Martino \\ DIMEC \\ University of Genoa \\ Italy
}

Submitted : 29 Sept 2021 ; Published : 26 Nov 2021

\begin{abstract}
The civilisation is amazing result, with impressive attainments to believe cosmic or godly reasons of it. The continuance, today, is critical, due to exhaustion of resources and contamination of environs, practised to keep the suited trend. The ecology review of the progress requires cognition and awareness of the advances ways, technologic and political aids. The sustainable ecology needs resorting to human intelligence, having integrated reliefs by cosmic rationality or godly wisdom. The robotic helps open to inner/upper causes, with steady issues.
\end{abstract}

\section{Keywords: CONSCIOUSNESS: COGNITION, AWARENESS - CIVILISATION: TECHNOLOGY, POLITICS SUSTAINABILITY: SAFETY, ROBOTICS - RECOVERY: STEADY, PASSING WAY.}

\section{Introduction}

The ecology is warning for the earth's inhabitants, saying that the extant over-exhaustion/contamination are frightening, leading to lack of sources and to poisoned environs, unless salvage measures grant retrieval duties. The civilisation self-ruin is odd choice: it advises looking at automatic trials, monitoring trends and fulfilling suited reclamation, as if inner/upper aids exist, provided by autonomous planning. The guess joins our trust in missions to perform and consciousness to be tiny fact, not altering universe goals. The mission is our civilisation, with scientific construal and behavioural principles; the consciousness brings to recognise that rationality or wisdom are absolute values, belonging to the all galactic context, while the humankind are just negligible part. The rescue entails our planet, minimal entity, in the huge universe, which cannot affect the galactic progression, only due to local downsides. The saving asks ding our habitat, not, just, on the individual comfort: the ecology protection is human collective concern, needing global prospects.

The ecology starts drastic changes in the humankind, modifying our truths in progress and growth. In old ages, the civilisation is variation of wild surrounds, towards safer and friendlier homes. The shifts identify progress, granting improved wellbeing to folks. Civilisation opposes to wilderness by culture and education. However, the beginning is not chaos: around us, the reality describes as cosmos, distribution of stuff, with coherent order: the details, namely, reveal, if we look at immanent information, cosmic rationality, or, if we trust in transcendent facts, godly wisdom. Thus, we interact with total truths, displaying absolute physical laws: matter and energy fields, depicted by causal models. The laws, detected by onlookers, are galactic information, namely: qualities, which define the transformations undergone by the reality; or spiritual facts specifying the said transformations. We feel interdepending with complex situations: we operate within our planet; the cosmos is backdrop, ruled by laws, spotted after experimental observations; it

The interactions with the environs shows that we are agentive and we enjoy cognition; it affects us, giving details on what happens around us. We ca modify course of events and activities; the observation of changes brings evidence of the galaxy, geode and anthropic spheres, with linked connections:

- at sidereal range, loose exchanges collect files and profit of the solar energy radiation;

- at earthy range, interactions affect atmosphere statistics, ecology caveat (earth warming), etc.;

- at anthropic range, contacts concern biology and cognition processes and linked facts.

The dealings are characterising data: material entities, linking effects and construal meanings. The items involve object of the reality, physical laws and agreed explanations; the material layouts have current laws and readings. The narrations benefits from identified actors with cognizance, widening the knowledge and allotting consciousness. The «cognition〉 and 〈knowledge avoid deterministic, with causal cogency: biology looks allotting self-ruling agentive talents; cognition appears adding thinking and judging abilities, enabled by observing the reality. The life self-acting and replication autonomy together with the cognition reasoning and decision making allow modifying the trends, promoting civilisation by innovation and planned welfare. The growth builds, using human intelligence to select improvements and to avoid snags, until the required exhaustion and pollution problems make dangerous the manmade processes; safe rescue implies: 
progress myth: reasoning and judgment are value added with no makeover hitches;

- ecology sustainability: reverse logistic allows full retrieval and fitting compensation.

The recue asks strictly involving incorporeal sources and performing circular conversions, without entropy piling up. The guess is men centred picture, founded the on biology/ cognition oddities; the cosmos centred trails assume that the earth's facts negligibly affect the universe. The local civilisation results have inner or upper origins, already included as galactic information; similar outcomes, most likely, happen elsewhere in the universe, with equivalent relevance. The biology/cognition oddities allow creating valid depictions and aware assessments of what perceives, by inventing interpersonal knowledge encoding; the earth case is, perhaps, singularity, or iterated even, showing that similar full readings and aware accounts repeat in the universe, providing visibility of cosmic rationality or heavenly wisdom as general aspects, not just human intelligence oddness. The hypothesis considers the rationality or wisdom, to be natural/spiritual aspects, already present in the reality and shared by the galactic ensemble. The alternate hypothesis is optimistic, maybe; its plausibility involves a series of aspects, starting from progress consciousness and civilisation deployments, the, covering the sustainability prerequisite and the recovery conditions. The topics request explicit specifics, summarising, in the following four sections, some recent personal ideas [1] - [16].

\section{Aware Headway}

The hypothesis of rational or wise reality leads to believe in coherent galactic laws, ruling the all, maybe, distinguishing natural from spiritual laws, if these are immanent or transcendent. The human intelligence is not earth's anomaly; in the universe, the agentive/cognition skills repeat, yielding «synthetic hands/minds〉, to make conscious improvement feasible. The guess leads to monism, with information (natural laws) inner aspect, or to dualism, with holy reality, located upper the matter. In the former, the inner sides play blind, deterministic roles; with forged monism, these qualities might be known (if detected and encrypted) and processed to aim at changes. In the latter, the upper sides is spiritual entity, with thinking and judgmental functions, supplying improving goals, by godly dualism. The forged monism and godly dualism enjoy inner or upper controls, prospecting intrinsic rubrics, already incorporated by the reality, or with holy origin.

The cognition (or thinking and judgmental function) qualifies third observers of facts and acts happening around them; the present account is widening construal and it distinguishes:

- human cognition: the process fulfils abstraction and encrypting of intangible knowledge〉;

- Robotic cognition: the process detects abstract qualities or spiritual entities, from the reality.

Our bottom up deductions avail bystanders: the human knowledge>, built by biology/cognition way, is intellectual construal, with self-sufficient description, however, the 〈knowledge〉 can equivalently specify as galactic series of attribute, already present in the reality and subsequently discovered by men:

- the human knowledge is contingent outcome of the abstraction/encrypting cognition;

- the absolute knowledge is spiritual evolution of what happening in the tangible reality;

- The implicit knowledge is aspect, replicating galactic information carried by the matter.

The abstraction/encrypting cognition is, just, human fantasy, interpreting currently acquired perceptions. By dualism, we have godly disclosures of the galactic information, readily available as absolute knowledge. By monism, the information is inherent quality, detected, in the forged version, together suited carrier that can have processing, as implicit knowledge: watching functions inspect the environs, making inferences and shared agreement. The analyses, unless believing in godly exposes, are trying, with queries, if contingency of thoughts and reasoning need transform in total truths. The intricacy of the all becomes higher by using the word «knowledge to label different concepts, as above done.

The confusion is manifest, when, in monism, the watcher disappears, as if the implicit knowledge repeats galactic information. Then, the aid offered by the biology/cognition procedure becomes puzzling, with third controls limited to inner rationality or to upper wisdom according to general principles, lacking inspections, if just a priori checks apply. However, suited faiths allow realistic choices, having validations purposes. The analyses shall go on using such viewpoint applied to 〈knowledge systems as feasible alternatives, to check the outlined conjectures. The «knowledge», as purposely noticed, is certainly human by-product; the extant (cognition〉 process entails the link of perception to symbols (abstraction), followed by managing the suited meaningfulness (encrypting) and the abstraction/encrypting sequence is intellectual accomplishment that remains at software levels, nether involving hardware modifications.

\section{BIOLOGY/COGNIZANCE WAY}

We mention together life and cognition, saying that we need hands (actuators) and minds (data encoders and processors): they are earth's singularity of the civilisation. They add selfactive hardware and planning software, with education and training (culture and ethic) consciousness. The knowledge supplies judgment and innovation. The exposure to and classing of feelings become shared experience, if the convergence on agreed marks occurs. All animals learn spotting and ranking and recognise symbols and scales, but men, in addition, select signs, with allotted meanings, to be useful as (spoken/written) semantic, describing what sensed. The human idioms, maybe, show wide fantasy, inventing many dialects and languages; computers, later, restrict to binary codes with added special symbols, allowing the translation to any other idioms. The abstraction-and-encrypting steps are enough to create collective understanding, maybe, starting with local (limited words) slangs and aiming at robot (universal dictionary) globalisation. The shared understanding, forcedly, limits to 
the involved communities; the presence of many languages aims at parting the peoples of the earth, at least, up to when a common comprehension could develop.

The biology describes life phenomena, once they exist, creating ordered sets of feelings or activities. The living organism enjoys agentive abilities: it follows lifecycles, keeping self-acting autonomy, from beginning (birth), to end (death). The agentive abilities are not obvious: biology shows odd upper/inner facts, as if all actions revel steering logics. Lately, the genome detection happens: encoded steps explain gene evolution plans. The spotting and ranking skills of men, availing of sophisticated imaginings and mimicry abilities, add exhaustive sound modulation and graphic encrypting options, leading to spoken/written languages for high effective communication. The thinking capabilities are not simple: cognition displays, maybe, the existence of upper/ inner causes, possibly, supporting the relational meds of communication, trade and authority. The meme fruition plans have software consistency; however, they may follow total inspiring controls. The hints suggest that biology and cognition are local anomalies, started by strange singularities. However, they can be, as well, total features, already ruled according to the extant galactic information. The two possibilities lead to wholly different prospects, say:

- the reliable development is bottom up contingent elaboration of the human intelligence;

- the progress is total attainment of the heavenly wisdom or the cosmic rationality.

With biology, the meme fruition assigns clever design competences, to select improvements and to avoid hitches, so that pace wise advances occur; the parallel view tells that presence of upper wisdom or inner rationality, in any cases, supplies suited backdrop, granting the civilisation. The former trail has dependent worth, with adaptive chances; the; both latter trails trust in absolute truths, looking at upper/inner ideas, as if absolute truths are available and suited attainments are always feasible. The biology and cognition events have start, perhaps, by galactic information: the related cosmic rationality or godly wisdom gives the wholly explanation of 〈synthetic hands/minds〉 already parts of the reality, not just earth's singularity.

\section{ALERT CONSCIOUSNESS}

The biology/cognition events consistently connect with contingent knowledge, due to human intelligence; they, further, prospect the mentioned links to cosmic rationality or heavenly wisdom. The analyses do not consider extra biology/ cognition events or possible other similar sequence since their existence is unknown and we do not have cues on how these new 〈knowledge systems〉 could organise. However, the analyses have to look at cognition functions and consciousness purposes with spread stability in the extant reality construal or authentication. The concepts behind «knowledge`, again, allow identifying meanings and roles:

- the intelligence offers spot selection for timely creating contingent human knowledge;

- the rationality supplies optimal way to get natural information, as intrinsic knowledge;

- the wisdom provides optimal pick identifying spiritual entities, as absolute knowledge.

The pictures identify observer to describe and appraise what tested around him; or they make resort to implicit knowledge and 〈big data〉 effects; or they avail of holy understanding, with absolute watching. The explicit monitoring clearly happens with onlookers; the assessments provide insights on what is useful or dangerous, to classify benefits or drawbacks and to acquire consciousness of how improving the situations. Witnesses plan the civilisation, with safe cognizance that advantage follow actions: the intelligence assures wright elections: the rationality or wisdom is absolute virtue, not asking on line checks. The monitoring and consciousness avail of men, robots or other 〈knowledge systems»; the robot way has to distinguishes men designed equipment, from inner/ upper driven gears, since explicit intelligence exists in in the first situation only, not just with rationality or wisdom. Indeed, the biology/cognition events are earth marks, but alike occurrences exist, bringing 〈knowledge systems〉 in galaxy spaces: 〈big data〉 and 〈big brother〉 are example robot-like total ways to deal with ecology sustainability problems, without explicit knowledge〉 handling. In current views, the conscious progress uses observers. The ecology, due to technology and political courses, justifies sustainability necessities, face to exhaustion and contamination impacts; the aware and explicit knowledge of the extant environs are must of actually performed measures: civilisation and sustainability imply cognition and awareness.

The 〈knowledge systems〉 are, today, vague concept: we know the earth human intelligence; the implicit or the spiritual knowledge requires faith that forged immanence or godly dualism offers direct (by cognition function) or holy (by spiritual means) access to meaningful encoding and reading of the galacticinformation. Monism rejects watching; the forged version reads and handles qualities with carriers, this brings to define implicit knowledge (tautology to label carrier-andquality), actually involving the timely picked carriers. The godly dualism implies transcendence; the factual version refers to the mind worlds, with the imagination replacing the absolute truths. The remarks tell that: forged monism handles carriers in lieu of the encoded messages, faking data flows; the godly dualism trusts in holy spheres; the factual dualism resorts to fantasy, to replace total truths, hoping in theories and trials to get validations.

The consciousness builds by the factual models, via cognition processes, building contingent knowledge. Monism/dualism way does not need open cognition; the knowledge) is abstract aspect or spiritual item, already present in the natural or spiritual reality and identified appropriate carriers or holy entities; these ways handle intrinsic knowledge, keeping implicit the all (not to infringe monism), or absolute knowledge, belonging to the holy reality (expression of dualism). The consciousness establishes by identifying courses, when detected the true values and agreed on the encrypted meanings; the cognition processes, here, need faith in the backdrop inner/upper origins. 


\section{IN PROGRESS TRAIL}

The aware civilisation, by biology/cognition way, avails of contingent knowledge, supplying technical and political innovations, to improve the affluence and wellbeing, exploiting natural resources and organising collective layouts. The progress is intelligent planning. By monism, Darwinism signifies optimal evolution, directly linked to the steering genetics by inner determinism, but intelligence needs decision freedom. By dualism, the wisdom selects ideal goals with consistent acknowledgment of holy truths, but intellect is far from certainties. The factual way follows bottom up courses: resort to fit technologies and behaviours; creation of governmental and administrative setups. The advances deploy pace wise, with steps ahead:

- technology revolutions, beginning novel production lines and efficiency goals.

- collective breakthroughs, originating political layouts and legal organisations.

The technologies establishing the empirical experience, looking at profitable trials, maybe, confirmed by theories. The factual dualism permits conceiving knowledge objectivity, keeping matter vs. information independence, in lieu of the determinism, which brings forth monism, merging the existing all together. The cognizance is event aimed at forming abstract duplication of the sensed impacts, transforming these in conventional symbols of the mind worlds. The codes are arbitrary, with clan's agreements and describe the reality by languages, assigning local effects. The 〈knowledge〉 objects are common reference, to find definitions and meanings shared into the different idioms.

The «knowledge $>$ objectivity is result of shared understanding, proving that plots and models have origin from the same things, acts or facts. The results authenticate abstraction and mind's worlds. The detected science happens rightly, identifying the galactic information. Positively, knowledge emerges with unbiased worth and autonomous truth, with collective agreement. Each person enjoys his natural talent, identifying the given mind, at this point, the description of suited natural and artificial intelligences is meaningful, as the mind's procedures have possible definition in. Life indorses agentive traits; cognizance gives intelligent charters. The cogency presumes coherence, i.e., explanation of events and facts, plus ability of consistent inferences. On the earth, by agentive and thinking behaviours, the changes look at improvements, with drifts thoughtfully chosen to address rational/wise goals, i.e., transformations enjoying of inner logic and upper dependability.

\section{TECHNICAL HEADWAY}

The progress is technical result, due to revolutions. In oldest ages, the manufacture of cloth and dresses and construction of edifices and houses covert men in garbed societies, inhabiting homes and towns; these archaic revolutions already distinguish mem from other animals. Then, the agrarian one aims at systematic resort to life processes, to multiply the available resources, by intentional farming by planned breeding and professional cultivation and husbandry. The industrial one looks at organised schedules, starting from the manufacture scientific planning; it knows variations, to switch at intelligent plans, to include robot aids and to widen the performed business to other technical areas. The planning of biology processes and efficient work scheduling are options, which show that the human intellect is self-governance processor, inventing tasks and means and programming arts and crafts; however, this does not entail self-sufficiency; maybe, inner or upper causes exist, directly ruled by galactic information and human knowledge is just mid help, making explicit the current attainments.

The knowledge formation and growth planning show conscious trails. The implicit galactic information gives selfrule to monism: entropy involves no growth sustainability. The upper steering allows autonomy to dualism: transcendence is faith, out of our spheres. The factual reading entails contingent truths, asking ecology checks. The technical headway opens queries. The aware planning is explicit, when dealing with contingent knowledge, since we find us, facing the query to solve; with absolute or intrinsic knowledge, the plans track assigned courses and our consciousness remains latent, with hidden decisions. Quick answers are not easy. The aware way follows clear cognition procedures, with abstraction and encrypting steps at interpersonal range. Noting what occurs around us, we distinguish existing different causes, which promote transformations, with upper/inner, human steering and new open/hidden singularities:

- spiritual/natural settings: variations promoted by unconscious upper/inner constraints;

- personal instruction: alterations develop by planned education and training of children;

- $\quad$ synthetic updating: changes due to physical laws plus stochastic singularities or miracles.

The cosmic/heavenly trails leave awareness queries, face to immanence/transcendence constraints. The human path enjoys explicit contingent knowledge; the synthetic way needs mention, as cognition processes shall repeat, due to universal rationality/wisdom. The knowledge of the technology revolutions shows clear dependences on inner/upper rules (manufacture modes, agrarian methods, etc.), to be part of the galactic information, already present as natural/spiritual backdrop of the human behaviour.

\section{POLITICAL HEADWAY}

The progress is political result, due to collective breakthroughs, developing civic/social orders in the earth populations. Spiritual settings, by dualism, assures explicit knowledge handling. Natural settings ask longer travels: biology appears being part of galactic information, the connected processes need singular starts, from which related modes are active. We do not know if this carbon-based life repeats somewhere in the universe or equivalent other occurrences is active. Additionally, the cognizance, imaginably, involves knowledge reading; still, the invention of abstraction and encoding starts formalising communication idioms, from which the relational 
modes permit building explanations of the observed reality, according to clan's patterns. The natural laws, on earth, include biology (genome's instructions) and the travel closes.

The singularity happen without apparent reasons, unless upper/ inner necessities assuring coherence to the extant universe. In this case, human intelligence is example attainment of inner/upper requisites, say, heavenly wisdom or cosmic rationality, enjoyed by the reality. If the human intelligence is origin of civic orders by boom up way, the backdrop truths exist and the top down way justifies the social orders, giving alternate deployments. The civic/social orders' equivalence is remarkable: the relational modes build three-range political setups, by communication (language), business (market) and authority (government) levels at the operative implementation. The executive layers materialise abstract principles, fostering contact, trade and headship, with stable worth and efficient administration features.

The political arrangements look at rational/wise planning, which bends determinism, with creation of governed nations and exploitation of clever transformation of extant sources. The men's history records series of collective breakthroughs from scattered clans, having no or negligible cross-coupled activities, to regular nation states, with self-rule split-sovereignty a, perhaps, to spread-out people, necessarily forming uniformly cooperating folks. The relational modes tells the families, clans and communities initially promote solidarity cohesion and rivalry protection. No other animals enjoy meme fruition tracks, using emulation/simulation, to devise the autonomous structures of administrative and governmental layouts, with legal sufficiency and interactive competition.

\section{SUSTAINABILITY GOALS}

The reality we know is physical aggregate of cosmic stuffs, exchanging fields and flows. It is quite credible that we also have galactic information, describing the all by coherent models. If we believe in monism, the narration shall see increasing entropy and residual waste, unless extra events happen, creating innovation, with aware recovery by forged monism courses. If believe in godly dualism, we shall attest the holy way or linked signs; the entropy affects the corporeal courses, but miracles can occur. Our science, in any case, expresses physical laws with entropy, availing of rules founded on causality. The astronomic sights seem offering highly unbalanced status of negligible planet, in unbounded universe: the former and related ecology trends will never affect the latter; in like time, any local component shares the entropy drifts of the overall universe, unless spot events occur or actors exist, creating unexpected peculiarities.

The extra events are possibility, which fulfils the basic galactic information. The biology/cognizance happenings are, perhaps, stochastic facts or holy miracles, starting earth's quirks to human intelligence, assumed spot marks, adding tof the cosmic rationality or heavenly wisdom. The outlined frames suggest that the contingent human knowledge may transform by covered or open details, using forged monism or godly dualism, into total knowledge. The detected physical laws are correct view of the reality. The ensuing civilisation combines technical revolutions and collective breakthroughs. The switch to total knowledge can entail robot-like aids or synthetic hands/minds, creating equivalent advances opposes to wilderness; these have direct support by universe for improved life-conditions. The progress is automatic pursue, written in the galactic rules. The description avails of the known absolute reference data:

- forged monism: the information is intrinsic quality of matter, detected with timely carriers;

- godly dualism: the spiritual reality rules all material courses, deliver at transcendence level.

The two tracks differ. The latter deals with explicit knowledge, belonging to the holy reality; the former handles implicit knowledge, to handle with (material) carriers. The immanence has to face the Gödel's theorem on the self-sufficiency of the axiomatic propositions.

- It is impossible to prove the non-contradictoriness of an axiom system by self-ruled guess.

- Fit mathematical algorisms show the lemma insistency by the system non-completeness.

The monism does not distinguish information from matter: this is tautology in the forged version: system completeness requires extra data. In conclusion: unifying logic coherence cannot have proof; for axiomatic definitions, the theory is defective at the range of scientific consistency of the founding completeness. The absolute (information) is faith, as the transcendence, out of matter, has faulty ontology proof. To conclude, by godly dualism, the autonomy of spiritual knowledge is faith; by forged monism, the knowledge handling us, as well, faith, built on fickle tautology. We accept that factual knowledge converts in spiritual or implicit data, developing scientific truths; the ontology evidences have doubtful theoretical foundation, because fit necessities due to logic absurdities are week truths, just, providing plausible guesses. The factual dualism is realistic estimation of instant qualities, allotted to current material supports, but the local picks have only contingent worth, not to be confused with absolute information. The forged monism or godly the dualism theories are worked out as believable models, to describe the physical reality around us and to explain the current transformations by total makeups. The theories are faiths or realistic accounts and, as such, they allow the following analyses, which keep the mentioned models, in the background.

\section{SAFETY AND CARE}

On earth, the ecology puts in discussion the life sustainability, because of resource exhaustion and waste pollution. The sustainability addresses the planet and the industrial competition policies of split-sovereign nation-states. The universe remain in the backdrop: the current changes are outside men's influences and we have little confidence on how unravelling our destiny from the galaxy, but we trust in its staidness. The planet's upkeep is warry, abruptly appearing in recent years, modifying progress myth and implicit safety. The depletion and disposal affect local snags, not universal; we may investigate the preservation or repair of the planet, in the galactic context, according to implied or direct lines: 
- the subsidiary aids explore the effects of the cosmic rationality or heavenly wisdom;

- the through helps look at supply and retrieval sources having interplanetary origin.

The maintenance and care of the earth require design and activity implementation by men or by robots, say, the execution of human works or the planning and effecting of jobs by inner/ upper bases. In all cases, the robots play data processing and task execution roles, which replace men to create or upkeep the suited friendliness of the surrounds by understanding current events and running apt plans. The industry 5〉 step describes the all, when robot-like procedures are in charge to synthetic hands/minds. This implied line has to operate with inner/upper instructions already present in the galactic information, which, perhaps, stat becoming operative after stochastic events or singularities. The linked occurrences have subsidiary label, since they just confirm the inverse's rationality/wisdom, parallel to the human agentive/cognition skills. The robot-like processes have galactic backdrop and we shall analyse them separately, since the conscious planning of the civilisation appear having unlike origins, even if the <industry 5> effects do not distinguish if ensuing men's design or already present byproduct.

The direct line is theoretically more problematic, because grounded on the human intelligence and on the linked contingent planning. What confined to happen on our earth is, surely, inconsequential, compared to the phenomena affecting the sidereal spaces and detected in interstellar space, unless the 〈knowledge〉 and the decisional awareness. The «knowledge〉 is men's construction, with contingent worth. The contrast of mind models and experimental cues permit acknowledging when results appear giving total assessments, factually, duplicating the (galactic informations. In that situation, the «physical laws〉 are available, bringing true worth and providing absolute 〈sciences by mental theories and experimental proofs, as investigations follow intellectual conjectures and tests. The parental scheme is operation way to conceive educational objectives and to implement organised societies with public structure and economic effectiveness. The all is appropriate for the political deployments.

The technical innovations require interacting with tangible stuffs. Mind metaphors (images and symbols) and relational practices (simulation and emulation) identify the <intellect> or intelligence), with parallel of occurrences and explanations. The developments need complex courses, with material changes and pace wise of principles and theories checks, testing the 〈progress> backed on:

- gene evolution, assuming natural betterment by adaptive generation of best-fitted species;

- meme fruition, looking after planned improvements, qualified by human intelligent choices.

The intellect) allows modifying the natural trends, because the men's agentive skill and dexterity allow selecting how addressing valid routes, while avoiding pitfalls. The men supply synthetic hands and minds, wits invented tools and designing aids. The 〈progress〉 is worth prospect, which proves that the men's shrewdness and expertise suggest aiming at advances and at escaping drawbacks, whenever free choice authorises taking profit from brainpower. The 〈biology> and «cognizance〉 grant outcome 〈awareness〉, with consciousness of the 〈ecology snags. The 〈progress picture is consistent with recue and sustainability, if the correct control on source lessening and waste corruption undergo safely controls.

The human 〈knowledge〉 is only abstract data or mind fantasy, not spiritual entity or tangible information: it does not give access to the holy reality, but it does not remain in the material reality (with 〈entropy)). The mere monism packs gen and meme processes: it may enjoy deterministic relationships. The forged monism, with gen/meme packing has information processing with entropy: the innovation, possibly, just, deals with implicit knowledge. The dualism separately deals with abstract or tangible flows. By godly dualism, 〈matter〉 and 〈spirit〉 are different realities, with own laws; the 〈entropy〉 affects the tangible courses, but «miracles〉 are possible. By factual dualism, the mental worlds are fantasy and the intellectual trails are mind courses: (cognizance), (progress〉 and 〈consciousness〉 can have planning at abstract levels.

\section{ROBOT-LIKE WAY}

The robot-like trails offer subsidiary aids to progress, if their planning exists in the cosmos rationality or in the godly wisdom. The gen/meme way is example singularity, aimed at local 〈cognition〉 and intelligence〉 options, to create aware 〈progress〉; the implicit/explicit 〈knowledge〉 role opens question, replaced by total galactic effects. The natural/spiritual perception becomes inner/upper outcome of in line facts:

- natural causation: immanent control applies, deciding out evolution upshots;

- holy lineage: spiritual rulings affects the heavenly courses, guiding the events.

The accounts move from men centred, to cosmos centred; the technical/political setups cannot progress by bottom up logics, but globalization and top down causes assign total layouts. These avail of science laws or of religions, with physical principles or holy backing and experimental tests or godly rules. From Newton on, the gravity's cosmology states the stars and planetary systems; then, Einstein general relativity unifies all force fields with Big Bang and Black Hole cues, lately empowered by gravity's waves. The transcendence, the other way, allows parting the reality, with upper absolute truths, offering self-explaining schemes.

Intrinsic rationality is axiomatic tautology of the cosmos, parallel to holy wisdom of the spirit. These faiths describe details, with deterministic galactic logic, parallel to godly miracles, but the theory self-sufficiency clashes against Gödel's theorem, as said. The absolute cosmos' data show that information does not avoid entropy growth, i.e., progress cannot continue or trends are not sustainable, as is universal constraint. The natural rationality or spiritual wisdom parallel and human intelligence typify the usual situations:

- belief in natural structural consistency of the universe and in liked rationality; 
- trust in upper coherent insight and in spiritual useful layouts to fix the reality;

- reliance in self-government and democracy, to enable local ruling authorities.

The robot-like way does not need explicit human enhancements and policies, replaced by supplemental determinism or judicious control, already provided by top down galactic information. Besides intelligence, by contingent (humanlike) aids, also extra rationality or wisdom, by total (robotlike) basics, support the progress, modifying wilderness, into civilisation. The total way fosters global uniformity, further than the regularity of the nation-state split-sovereignty: the local ranges start by multiple dialects and nation idioms; the global range enjoys of standard computer encryptions. The formers are bottom up results; the latter is top down inner/ upper setting, already fixed by the galactic information. The globalisation, recently, marks the total typifying situations, face to the contingent one: the natural rationality and physical laws or the spiritual wisdom and religious doctrines sanction absolute truths and behaviours.

The robots do not need building natural/spiritual principles: their «cognizance〉 is original function, never asking changes and local versions. The 〈awareness', as well, is existing purpose, merging folks and Decreed Rules: the uniformity is intrinsic fact: it establishes cross-border markets and worldwide contact; it avoids marginal dialects and talks, supported by meme fruition. The bottom up earth's civilisation is typical issue; the top down uniformity, as well, is standard possibility: the uniform imperialism (China) enjoys millennial stability; the parting into hierarchic setups (Europe) needs spot enhancements, being the neighbouring site lower class worlds. The latter scenario has city-states and nation-states; it modifies by contingent drivers. The former require strong inner/upper rulers or imperative motivations such as the coming ecology hassles: the two reasons can unify and the globalisation becomes sustainability necessity.

The mentioned typical situations are exclusive: the robots fitly replace men by synthetic hands and minds, meaning with it, that (agentive〉 and (cognitive〉 skills provide the way to conceive and to fulfil the changes, from wilderness, to the civilisation. The backdrop material universe shall possess the base prerequisites, in views to the example situations. On our earth, the third situation clearly appears; the other two are guesses only, if we have faith in ordered coherence of the galactic stuffs. Moreover, that appearance looks faulty, without permanent stability and, today, the ecology shows that we approach critical disorders, making essential the analyses of the alternative situations, for possible ways out. The investigation proposes suited cues, with robots fittingly replacing men, on condition that further criticalities can have correct answer. The enquiry shall move in such direction, combining (sustainability) measures, face to the <ecology snags.

\section{VIABLE RECOVERY}

The <ecology) is new defy, recently, appeared to the earth's inhabitants, face to the lessening of resources and adulteration of environs, affecting the entire globe, due the effectiveness of the industrial economy of the extant split-sovereign notionstates. The <ecology/economy dilemma shows the mismatch of technical advances and political setups: the ecology entails globalisation for recycle/recovery purposes; the economy asks parting, with the hope to shield the local advantages. The selfishness typifies regular closed societies, while altruism grants the top down complere political steadiness of the «global villages. The rescue is test to come: uniformity is mandatory need, to yield active balance regress and progress, the earth over, averaging planned projects' falloffs. The results follow alternate relational modes, which look showing:

- globalism, as if steered by previously encoded cosmic rationality or godly wisdom;

- localism, as if pace wise created by bottom conceived human intelligence choices.

The latter supplies passing results, not granting permanent attainments; the former assumes that steady intents exist. We leave undecided the dilemma, evaluating both issues. The global uniformity or the local regularity have relational modes, to implement social links and political setups: worldwide web and global village or home economy and nation-states. In like degree, the peoples require monitoring and regaining duties. The exhaustion/contamination control is realistic, if integrated watching/mending and real-time devices have direct enabling. The all routines have explicit fulfilment, under human planning; they can have robot execution under galactic instruction, with or without man supervision. The technology innovations can establish along similar trends. The execution routines and technical advances are standard options. The social structures and political setups show that a novel «collective breakthrough) is approaching the (global village〉 denotes the inconsistency of localism barriers at communication, market and governance levels. The legal layouts, as well, shall modify, inventing social organisations and political systems adapted to the management of individuals and collectives in the new living conditions.

In the course of the analyses on what happening on our planet, the processing of materials and gears removes sources and leaves trashes: affluence builds on feasting; sustainability needs controlled feeding. Levelheadedness asks essential tweaks, by basic descriptive models:

- 〈mere monism»: progress/regress is implicit happening, without on looking witnessing;

- 〈godly dualism»: tangible transformations and miracles have holy ruling and overseeing;

- 〈forged monism : information qualifies matter and can be implicit construal for events;

- 〈factual dualism〉: human 〈knowledge〉 describes the watched tangible transformations.

The descriptions and explanations require bystanders, i.e., are functions distinct from the matter, based on the abstraction of concepts, understood via encrypted formats. The conceptualising and formatting are human steps for «cognition> purposes; the human «knowledge is, possibly, only, example procedure to extract ideas and accounts. The overall galactic system can exist and evolve without narrations, making fully consistent the (mere monism), perhaps, only linked to the 
〈factual dualism〉, where the 〈knowledge〉 is just intellectual fantasy, with debatable or phony consistency. Instead, we further devise: 〈godly dualism), with the parallel heavenly reality and 〈forged monism), with information to qualify focused matter items. These definitions allow switching the human «knowledge〉, from contingent, to total feature, according to the up to now discussed derails. Summing up, the four models are relevant interpretation aids to designate 〈cognition〉 functions and «knowledge〉 procedures, with contingent and total worth, necessary to formulate viable recovery policies, face to resource lessening and waste piling up.

\section{STEADY INTENTS}

The 〈global village` and total policies happen to be consistent issue of (godly dualism) or (forged monism), when sgalactic information > is available by holy revealing of absolute «knowledge` or intrinsic recognition of implicit «knowledge». The ways are happy events or fit confidences, as already argued, once we trust in the spiritual reality for the intangible ruling of the material surrounds, or in the inborn natural consistency of the measureable galactic phenomena. These dependable faiths are applicable findings, if the spiritual flows suitably supply inspirations, inventions, intuitions and discoveries about what happens around us, or if the natural courses reliably prospect interpretations, explanations, reasons and motives of the current events. The existence of transcendent presiding or of immanent management is credible hypothesis, as soon as we understand that the earth's acts and facts are wholly thrifty events in the galactic system: we correctly shall recognise that the reality has proper actuality and evolution, completely unaffected by the human presence or absence. The (above said) faulty axiomatic proofs of godly existence or of cognition meaningfulness are, notably, minor details, face to the reasonableness of spotting total portrayals of our surrounds.

The decided definitions have reliable worth: the creativity is necessary for innovations and advances; the awareness is essential for controlled feeding and recovery. The absolute cognition may resort to top down enlightening or to inherent recognition; the consciousness connects agreed concepts and physical details. The cognizance develops on earth, giving the encrypted account telling the cosmos' order: genome shows biology processes; cognizance offers links justifying our ideas on how the environs modify, under natural or planned actions. The ideas, create by cognizance, link to galactic information, if the said access to absolute or implicit «knowledge〉 occurs, according the abstraction-and-encrypting procedures or similar identifying methods, with approved formats. The conscious selection of benefits and rejection of hitches needs judging and self-acting autonomy, anomalies of humankind, which endows thinking and decision-making. These are singularities, in the physical earth's backdrop, stating life and intelligence, but alternatives are also possible, by natural or spiritual means, or monism/dualism way.

As far as we can figure out, the singularities are explicit or not. The clear biology/cognition pair generates animals and men, but we cannot excluding different other local actuation events or information processing results (〈big data〉 and 〈big brother〉 effects). The gene/meme evolution, cognition by parental education, etc., are open example courses, but alternate practices may exist, to implement knowledge, mental pieces of culture and ethics, or equivalent other formatted messages, having unrestricted worth. Without explicit singularities, the material processes and the linked attributes or qualities do not transform in knowledge», mental pieces or other encrypted figures/words. By 〈mere monism〉, of course, the reality follows any path without observed tracking; the picture usually connects to determinism, without innovation and progress, even if it is impossible acknowledging the factual sequence of facts. The occurrence of singularities, as well, can haver or not formal evidence. However, to conclude the topic, the explicit «knowledge entails handling spoken/written symbols, having previous effecting of abstraction and encrypting operations. The listed four descriptive models depend on the «knowledge〉 statement, but implicit way is hidden report. The cognition offers apt skills to human people: the knnowledge handling shapes minds and intelligence.

The instruction starts at parental rank (meme fruition) and it include lifelong conscious learning: it handles explicit software: the term replaces (godly dualism) absolute or (forged monism) implicit (knowledge». The word soundly deals with monism, when the forged version explicitly addresses software (not hardware). In robotics, the perfections started with designing programmed actuation devices, to replace men: today, the robots include data processing equipment managing automation or regulation apparatuses, as well, doing administration, business, service, etc., duties. The robots, in synthesis, appear being sets of instructions or programmed rigs, supplying synthetic hands and minds for clever planning. The gears include computer aids and other information processing tackles, carrying 〈big data〉 and 〈big brother〉 effects: the behind planning reflects decisional capabilities and it does not simply marks deterministic trends. The instructions (data links to planning) add adapted changes and intentional ticks: the variations are, possibly, optimistic fantasy, with hypothetical foundations.

The 〈knowledge seems to be essential, basically, for the progress planning and, presently, for the ecology sustainability of the plans. The data handling describes the build-up of 〈knowledge): this links to absolute or implicit knowledge〉, marks of original heavenly wisdom or natural rationality, adding to switch wilderness to civilisation. The progress, indeed, shall track a long ways. Gene-evolution happens modifying the genome data, leading to new varieties, species and classes; the set of «singularities) shows the <biology/ cognition> track of changes on our planet, along which the men direct inventions are limited steps, with 〈relational〉 labels, accepted as shaping interpersonal symbols. The slipup of absolute, implicit and human «knowledge` entails hardware/ software catch: spiritual reality, quality autonomy and mental fantasy are puzzling facts. The bottom up identification of the cultural discoveries and social architectures of the civilisation 
builds political deployments and technology revolutions. The bullied knowledge, if contingent mental construal, does not allow reaching permanent truths. If, instead, total knowledge is available, actual outcomes will endure stable worth and sustainable prospects, depicted by top down references. In the former conditions, the transparency of reasoning and decision planning wholly defines at human levels; in the latter ones, the heavenly/cosmic biasing overlaps, widening the promotion and responsibility ranks.

The top down ruling makes the individual free will questionable, mixing the paths backed by inner/upper causes, say, the deterministic/heavenly drifts of the universe. The merger implies that immanent rationality or transcendent wisdom automatically applies; the mix allots total progression. The 〈knowledge〉, perhaps, implicit quality or absolute entity, provides the awareness of the current conditions, showing the changes undergone by the surrounds, ecology hitches included and the clever deployments given by the progress. The civilisation becomes combined issue, with explicit, implicit and absolute 〈knowledge»:

- the explicit input adopts human intelligence management, moving from contingency worth;

- the implicit or absolute input directly applies total immanent rationality or transcendent wisdom.

The top down combined options enjoys inner/upper inputs, inspiring the mental choices: decisive actions follow with widened capabilities: planned tasks and clever grasps need acts and decisions, driven by total schedules, having previous coding or on line conception. In the study, 〈robot's path〉 alternatives happen, referred to 〈forged monism〉 or 〈godly dualism〉. Suited cosmic or heavenly utilities, possibly, rule «synthetic hands and minds», having 〈big brother management. The robot-like alternatives offer critical chance, when the ecology sustainability of the progress asks impending imperatives.

\section{PASSING ISSUES}

The 〈global village> is impressive collective breakthrough, replacing the «nation-state〉 regularity, by the open society uniformity. Presently, several marks prospect the globalisation, but other signs tell that the uniformity is utopia and the earlier localism keeps sounded foundations:

- worldwide web: the individuals differ due to native language, cultural/professional education, etc.;

- duty-free trade: the markets depend on local climate, agriculture, logistic, civic administration, etc.;

- global village: the citizens enjoy modifiable legal, economic, social, etc. positions and political rights.

The 〈relational〉 trail develops on folks, assembling people of different age, intelligence, fitness, etc. and bringing forth dissimilar necessities and interests. The uniformity is unnatural and, as such, it is meaningless ideal; the communal regularity seems telling ethnic variety and mental diversity logics. The 〈global village〉 is epitome of homogeneity, avoiding the diversified grouping. In reality, imagery and allegory are practice: the devised meanings at communal worth, defining contingent truths. All depictions are arbitrary; then, the local communities propagate ideas by instruction and training.
The mind metaphors (images and symbols) and interpersonal practices (simulation and emulation) appear with men, outside biology. The symbols are abstract picks, without link to absolute facts. The existing languages follow depending procedure, ending by outcomes with provisional worth. The settled 〈cognizance〉 and 〈knowledge〉 need shared agreement, to be meaningful, within closed societies. The selected computer instructions have global consent: this does not give absolute truth. That 〈knowledge〉 contingency 〈godly dualism〉 weakness.

The bottom up localism has factual suitability, without involving original truths. The human 〈knowledge is only encoded data, on which the minds play; the brain deals with the data carriers. The switching to total truths avails of theories and empirical proofs, turning conjectures of factual, in faith of godly dualism, with absolute knowledge in the natural laws and keeping the parting of mind's worlds by abstraction. The forged monism avails o intrinsic detection of galactic information, which leads to implied cognition, but allowing the mind-detected data (or brain-sensed hardware) and leading to implied cognition. The mind's worlds, yet, limit to (factual dualism〉 management, with reasonable links to the other inferences, bringing only to software identification: the axiomatic tautologies just exist for the ontology proof of transcendence and for the intrinsic detection of data. The human «knowledge〉 building combines imagery and allegory, as meme fruition resorts to simulation and emulation. The 〈relational〉 frame is mental creation, active when people interact according to communal conventions.

Without globalisation, the 〈factual dualism〉 only manages passing issues. The «cognizance has no links with the «galactic information>, unless theories and experimental checks could suggest models and laws, transforming contingent ideas, in absolute truths. These conversions have from the life/ cognizance start; they move through parental transmission, linking data in causal sequences, to make forecasts possible. The civilisation is outcome of mindful planning, based on conscious selection of the tasks to accomplish. In men, predictions resort to intelligence: shared agreement on encoded concepts establishes backdrop science, on which developing technologies and politics for the progress. The contingency way seems self-ruling, eluding the access to galactic information; the cognizance by interpersonal statements, built on the interpretation of the surrounds, enjoying shared conventions. The cosmic rationality or godly wisdom offers total routes, avoiding cognizance and awareness, when the changes have directly govern by inner/upper instructions, thus yelling deterministic progress (with no need of aware planning). The previous point joins the 〈human intelligences with 〈cosmic rationality〉 or 〈heavenly wisdom»; the combined ruling shows robotic awareness and it can classify as steady option. The present point leaves out reaching true information; without total 〈knowledge», the conscious planning can only look at passing issues, within bounded horizons.

The 〈worldwide web〉 and 〈duty-free trade〉 without 〈global village> are practical option, readily studied. The political 
localism preserves the 〈nation-states〉. The 〈relational modes〉 need altering the sovereignty and including the global 〈ecology) constraints. Again, what is happening on the earth is wholly negligible for the sidereal space: thereafter, also we do does not affect the universe: the existence or disappearance of the life/cognizance singularities is without outcomes. In like time, if men plan provisioning and sanitising policies, taking interstellar energy and stuffs, the actually produced upshots shall not modify the galactic course of events. The men's extra-terrestrial missions and activities are chance to be spent, because our cognition and agentive abilities allow profiting of the interplanetary discrepancy, without altering extant equilibria. Along with contingency visions, the globalisation offers highly different prospects: we do not, any more, hope the somehow share the cosmic rationality or godly wisdom benefits, rather to exploit the huge disparity, to get something at no costs.

The earth's peculiarities look more to localism, rather to globalism. The actuation abilities of the life have gene reading and possess gene evolution features that show how diversified are the natural outcomes of biology. The life is complex occurrence, whose diffused falloffs are vegetal or animal species, with riggings at local progressions. The cognition abilities of human minds have meme interpretation and have emulation and simulation practices that document how copying and reproduction allow describing what sensed and leaving accounts of transformations and histories. The 〈actuation〉 and «knowledge〉 anomalies have spot effectiveness, linked to instant acts and local details. The gene and meme explications do not point towards rationality or wisdom; their aim at intelligence limits to the choice among (inter legere) substitutes. Then, the passing issues are realistic acknowledgment of current facts: the other fancy ideas are guesses.

The mention of these end deductions is relevant: we cannot ignore the extant faith in the shared science and physical laws, today, exploited as absolute 〈knowledge〉 and total truth. The consequent inferences and judgments are successfully used; they do not remain fancy guesses (compulsory, lacking the faith in 〈forged monism〉 or 〈godly dualism〉). If just 〈factual dualism > is applicable, the resulting inferences and judgments could not have reliability. Therefore, the above end deductions happen providing also optimistic readings. Even remaining at contingent worth, the human 〈knowledge〉 of the shared science grants efficient upshots; the extra-terrestrial missions and interplanetary exploitations can modify the sustainable ecology forecasts. If correct, the (global village〉, as well, become hint for compulsory cooperation plans, at practical ranks.

\section{Conclusion}

The ecology warnings are critical prospects: they make unbearable the civilisation continuance, keeping the extant resource spoilage and pollution increasing. The <industry 5> step suitably defines the compulsory technical changes, listing robot-like duties, which need having automatic implementation, to keep fit supply safety and waste control. The 〈global village〉 is hypothetical new political setup, with societal uniformity of the devised cooperating peoples. The sceneries are not without ambiguities, according to the ideas, hinted by the study: men and robots can merge or alternate; globalisation can be (collective breakthrough) or not. The aspects show continuance vs. switching catches: they oblige deepening the globalisation consistency, here, notably, described as if total «cognition〉 backdrops make assuming the different robot-like roles and allot consciousness of the undergone transformations. The present enquiry shows the extant haziness:

- 〈cognition»: the process of specifying symbolic classification for all the sensed perceptions;

- 〈knowledge»: the human readings of the galactic information and linked relational setting.

The elucidations are necessary to specify the continuance vs. switching catches, not, just, ecology. In view of continuance, the 〈industry 5〉 step is right answer about ecology, by technical kits and big political needs. At first, 〈ecology〉 and 〈economy〉 seem having similar aims, say, the homes directly sheltering the men. Yet, the latter aims at bottom up budget and the former at top down views: by no more habitable $r$ homes, our survival vanishes; rescue and safety need looking at the human habitats, as vital bid. The technical changes implies a political breakthrough: worldwide citizens interact into open bonds, avoiding biased selfishness of rival kinsfolks; contacts have earth's range, trade aims at abolishing customs or tolls, acquaintances replace foreigners. The single (global village〉 unifies many self-governing nation-states. Duties and levies, actually, are official ways, managed by sovereign authorities to distinguish local folks; the governmental borders are sheltered tools, granting profits of efficient powerful countries.

The split-sovereignty of many fatherlands has long history, becoming assessed truth and placing in utopia the global village>. The multiple homelands have proof on racial, ethnic or cultural bases and keep efficacy on political, economic and financial viability. The legal setups of the local sovereignty have agreed statutes; their natural or godly origins deserve proof, unless their faith acceptance is all right. We have listed series of technology revolutions and collective breakthroughs, ending by globalisation; this last can be the start of the sglobal village and the end of split-sovereignty legitimation. Back to the extant haziness of the enquiry, we need be back to the «cognition〉 elucidations, specifying the continuance/switching catches.

If the 〈knowledge〉 is just men's invention, it cannot affect the reality and the hybrid scenarios, linking to the galactic information of the investigations, are out of reach. Our trust in the discovered physical laws is the way to faith in galactic information, thus, leaving out the «mere monism〉, to the 〈godly dualism〉 or the 〈forged monism〉. Thereafter, 〈cognition〉 can be spiritual or natural truth, enabled by singularities, as it is the case, on the earth, together with 〈biology»; the narration and judgement of facts are parallel events of the reality, as absolute spiritual reality or implicit natural quality of matter. The duplication with absolute 〈knowledge〉 or the detection with implicit〈knowledge〉 are plausible collective 
results, after experimental tests, possible, if the galactic information is original entity (dualism) or quality (monism). The specification of galactic information, spiritual entity or material trait, is complex affair, with access to transcend spheres or identification of immanent features: the personal 〈cognition〉 follows subjective abstraction/encrypting choices; the total specifications relate to spiritual facts or natural aspects, already fully stated. How these personal choices can transform in absolute information is amazing: if the discovered physical laws correctly model our environs, the astonishment authorises the faith in science and technology.

The 〈factual dualism> limits to the mind's worlds, managing perceptions, ideas and judgments and leading to theories and models, for inferences and forecasts. The mental spheres are immaterial because imagined, but not transcendent: the factual dualism replaces spiritual reality, by intellectual thought; the contingent fantasy avails collective reasoning, experiential testing and inventive intuition, to figure out absolute truths of the 〈godly dualism〉. The alternate 〈forged monism〉 presumes intrinsic 〈cognition〉, avoiding fantasy by direct detection of the matter qualities: the issues are data handled with carriers, by software identification on subsidiary hardware. As evoked, axiomatic tautologies exist for the ontology proof of transcendence and for the intrinsic detection of carried data: we cannot use the axiomatic definitions, as proofs of the lemma. The existence of spirituality transcendence of intangible thinking/designing or the identification of natural immanence of qualifying information happened to be credible hypothesis, justifying human 〈knowledge〉 conversion in galactic information, if trustful premises, godly wisdom or cosmic rationality justifies what done by human intelligence, within globalisation prospects. The enquiry imagines that the 〈global village is feasible and the suited upper/inner-ruled supply/depollution chances are reasonably rooted on total views. In shared beliefs, scientific theories and physical laws are truths, with coherence: the foundations happen being the transcendent or immanent galactic information, according to usual readings.

The interpretations look at upper wisdom or at inner rationality, putting the cogency in holy or in natural reasons, already stated at the galactic ranges: the terrestrial events and human dealings have to follow the extant directives, since our intelligence) and 〈knowledge〉, possibly, modify derails, not surely the general trends. The spiritual construal envisages godly spheres out of the tangible reach; the natural one considers intrinsic restraints within the material context; the belief or envision of wise religions or rational coherence has, perhaps, the evidence of monitored facts and acts, but the a priori assumption is faith. The a posteriori construal gives plausibility to the faiths, even if it is non-sufficient explanation of the human civilisation. The enquiry enchain cognition-consciousness-planning, to grant progress, since we need on looking and judging capabilities, performing parallel material trifurcations and acknowledgment tasks, leading to:

- the spiritual/natural settings accomplish changes by unconscious upper/inner makeovers;
- the personal programmes implement advance, after fit instruction, tests and corrections;

- the synthetic designers/actors look at progress by deterministic trends and aware control.

The upper wisdom or inner rationality are meaningless, without 〈knowledge〉. The aware way follows knowledge definition: human cognition is clear possibility, having contingent upshots; these are total, with upper/inner aids. The last line can have start by open/hidden singularities in the galactic information, which add localism effects, such as life and intelligence or others. Carbon-bases biology and abstraction/encoding cognition are earthly peculiarities, maybe, locally replicated, to reach similar effects, by different methods. The wisdom/rationality qualities are odd results, to spread over the universe as galactic information, not figured out as spot competency, perhaps, locally promoted by stochastic singularities. The conclusion of the enquiry shall ignore the first line, no involving the human intelligence. It shall discuss, along the second line, the aspects defined passing issues, to find out possible ways to steady targets. It, purposely, shall focus the attention, along the third line, on the cognition and consciousness aids, leading to the 〈global village setup after the globalisation breakthrough. The enquiry keeps centrality on the knowledge〉, to enable the aware headway, looking at diversified sources and performing decontamination tasks. The ecology sustainability seems requiring provisions exceeding the men's capabilities, even via extra-terrestrial missions; the viable recovery needs resorting the total «knowledge», thus, to upper/inner aids, already provided by holy/natural ways. The 〈godly dualism〉 or 〈forged monism> model entails the transcendence or immanence criticalities, extensively examined by the present investigation.

\section{References}

1. Michelini di San Martino R.C., Ed (1976) XIV BIAS: Automation and resource utilisation, FAST, Milano, p. xv-476.

2. Michelini di San Martino R.C (2008) Knowledge entrepreneurship and sustainable growth, Nova Sci., Hauppauge, p. xviii-325.

3. Michelini di San Martino R.C. (2009) Robot-age knowledge changeover, Nova Sci., Hauppauge, p. xvi344.

4. Michelini di San Martino R.C (2016) Cognitive revolution quest: human civilisation prospects, Aracne, Roma, p. vI357.

5. Michelini di San Martino R.C. (2017) The relational trails to sustainability, Intl. J. Sustainable Entrepreneurship and Corporate Social Responsibility (IJSECSR), vol. 2(1): 141-155.

6. Belotti V., Michelini R.C., Razzoli R.P (2017) Relational modes and thrifty progress, Int. J. Application Innovation in Engineering \& Management, 7(1): 131-146.

7. Michelini di San Martino R.C (2018) Civilisation modes and ecology constraints, Intl. J. Historical Archaeology \& Anthropological Sciences, 3(6): 803-812.

8. Michelini di San Martino R.C. (2018) Business sustainability and frugal chances, Int. J. of robotic engineeing, 2(5): 1-11. 
9. Michelini di San Martino R.C (2018) Progress bases: biology and cognition, Intl. Robotics \& Automation J., 4(6): 358-366.

10. Michelini di San Martino R.C (2019) Humankind engineering and management: robotic track, Journal of Robotics and Automation, 3(1): 83-98.

11. Michelini di San Martino R.C (2020) Anthropic advances: global robot driven rescue, Int. J. of robotic engineeing, 5(1): $1-15$.

12. Michelini di San Martino R.C (2020) Manufacture paths: robotics \& sustainabilty, in M.A. Mellal, Ed., Advanced Manufacturing: Progress, Trends and Challenges, Nova Sci., New York, chap. 7, 126-188.

13. Michelini di San Martino R.C (2020) Progress queries and sustainability: basic views, Intl. J. Clinical Studies \& Medical Cases, 6(4): 21-29.

14. Michelini di San Martino R.C (2021) Manufacture/ relational modes: robotic sustainability, Intl. J. Research Studies in Science, Engineering \& Technology, 6(1): 9-22.

15. Michelini di San Martino R.C (2021) Progress management and ecology sustainability, Int. J. of Medical and Clinical Studies, 4(3): 1-15, ISSN 2692-5877, ijclinmedcasereports.com.

16. Michelini di San Martino R.C (2021) Thrifty hedaway: Robot cooperative aids, Client Periodic Quarterly Medicine, CPQMe, 12(3): 1-29.

17. Michelini di San Martino R.C., 201, The robot awareness sustaunability, Intl. J. Education, Business \& Economics Research IJEBER, vol. 1, n. 1, Nov., pp, 49-71, 1 IJEBER www.ijeber.com.

18. Michelini di San Martino R.C., 2022, The relational hypothesis of uuman societies, Biomedical Jornal of Scientific \& Technical Research, vol. 40, n. 1, pp. 1-18, ISSN 25741241.

Copyright: C2021 RINALDO C. MICHELINI. This is an open-access article distributed under the terms of the Creative Commons Attribution License, which permits unrestricted use, distribution, and reproduction in anymedium, provided the original author and source are credited. 